\section{KOMPASS}

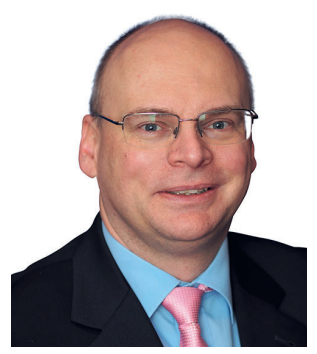

Peter Heinz

Augenarztpraxis Dr. med. Peter Heinz,

Schlüsselfeld, Deutschland

Die deutsche Augenärzteschaft versorgt die Bevölkerung, trotz aller systembedingten regulatorischen Widrigkeiten, auf die ich hier nicht weiter eingehen möchte, in der Regel gemäß den aktuellsten Erkenntnissen der modernen Augenheilkunde. Leider hat die OVIS-Studie (Ophthalmologische Versorgung in Seniorenheimen) der Stiftung Auge gezeigt, dass die ophthalmologische Versorgung sich mit dem Umzug in eine Senioren- oder Pflegeeinrichtung schlagartig verschlechtert. Dies hat teilweise erhebliche Folgen, sowohl für die betroffenen Senioren als auch für die Einrichtungen, und verursacht letzten Endes auch erhebliche vermeidbare Folgekosten.

Es ist unbestritten, dass schlechtes Sehen die Sturzhäufigkeit, mit all den verbundenen möglichen Komplikationen bis hin zum Tod, massiv erhöht. Von den daraus resultierenden höheren Pflegekosten ganz zu schweigen. Auch die kognitive Leistungsfähigkeit hängt mit einem guten Sehen zusammen. Nicht zuletzt ist das Sehen für Lebensqualität im Alter ein - wenn nicht sogar der - entscheidende Parameter.

Sind nicht die Versorgung und der Umgang mit unseren Seniorinnen und Senioren ein wichtiges Kriterium für die Menschlichkeit und die «Caritas», die wir unseren «Alten» entgegenbringen? Hierzu hat die Stiftung Auge diverse Maßnahmen angemahnt und

\title{
Augenärztliche Versorgung von Seniorinnen und Senioren: Ein Kriterium für die Menschlichkeit
}

es muss sich zeigen, ob bei den Entscheidungsträgern in Politik und bei den Kostenträgern wirklich der Wille besteht, hier zeitnah Verbesserungen zu erreichen. Eine einfach umzusetzende Erleichterung wäre die generelle Übernahme der Fahrtkosten von Bewohnern der Alten- und Pflegeeinrichtungen in die augenärztlichen Praxen, auch wenn es sich nicht um stationsersetzende Behandlungen oder Leistungen handelt. Auch die intensivere augenärztliche Betreuung dieser Patienten sollte sich im Honorar niederschlagen. Die Forderungen liegen auf dem Tisch und wir dürfen gespannt sein, was sich hier entwickeln wird.

In der Übersichtsarbeit dieser Ausgabe gehen die norwegischen Autoren Brunes, Hansen und Heir aus Oslo der Frage nach, wie sich schlechtes Sehen bei Erwachsenen auf die soziale Integration und Interaktion auswirken. Es zeigte sich eindeutig, dass schlechtes Sehen allgemein mit einem erhöhten Risiko an Einsamkeit zu leiden einhergeht. Diese Entwicklung ist gerade auch für Seniorinnen und Senioren fatal, da Vereinsamung natürlich die Lebensqualität enorm verschlechtert.

Ein Bestandteil des Älterwerdens ist die Presbyopie. Aber um das KAMRA-Inlay ist es wieder sehr still geworden. Björn Bachmann (Köln) stellt eine der Ursachen für den fehlenden Erfolg dar.
Im Fallbericht berichtet eine spanische Gruppe (Barcelona) bestehend aus Tellez, Vela, Luna und Delgado über einen 80-jährigen Patienten mit proliferativer diabetischer Retinopathie und Sekundärglaukom, bei dem es nach einer Vitrektomie in Kombination mit einer zusätzlich erfolgten Implantation eines Ahmed-Gonioimplantats zu einer massiven Migration von Silikonöl in den subkonjunktivalen Raum kam. Es werden diverse Pathomechanismen diskutiert. Anhand dieses Falles wird ebenfalls deutlich, wie wichtig eine funktionierende augenärztliche wohnortnahe Betreuung unserer Patienten - auch denen in Alten- und Pflegeheimen - ist, da im Falle von komplexen Operationen eine optimale Nachbehandlung unumgänglich ist. Sonst bringen möglicherweise alle (kostenintensiven) operativen Maßnahmen keinen Erfolg und Gelder wurden somit wieder «in den Sand gesetzt». Unser Gesundheitssystem sollte nicht an den falschen Stellen sparen. Ich wünsche Ihnen viel Spaß bei der Lektüre.

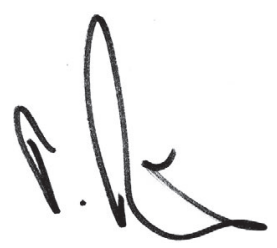

Peter Heinz

\section{KARGER}

Fax +497614520714 information@karger.com www.karger.com
() 2019 S. Karger GmbH, Freiburg

Accessible online at: www.karger.com/kop
Dr. Peter Heinz

Augenarztpraxis Dr. med. Peter Heinz

Bamberger Straße 32

96132 Schlüsselfeld, Deutschland

heinz@augeninfo.de 